\title{
On the Theory of Scalar Diffraction and Its Application to the Prolate Spheroid*
}

\author{
N. D. Kazarinoff and R. K. Ritt \\ Department of Mathematics and The Radiation Laboratory of the Department of Electrical \\ Engineering, The University of Michigan, Ann Arbor, Michigan
}

\begin{abstract}
Scalar scattering of a plane wave by a perfectly reflecting body whose surface is a level surface in a coordinate system in which the scalar wave equation is separable is considered. A general method for the computation of the surface distribution is described. This method reduces the problem of finding the surface distribution to that of evaluating a certain contour integral. The distribution induced on a prolate spheroid by an axially-symmetric plane wave is specifically computed. The evaluation by residues of the contour integral, given by the general theory, leads to the expected "creeping wave" interpretation of the residue series in which the attenuation of the "creeping waves" depends, in first approximation, on the local radius of curvature. The asymptotic theory used is applicable for large values of $c \omega$, where $2 c$ is the interfocal distance of the spheroid and $\omega$ is the wave number. The surface distribution is computed over the entire shadow region including the tip.
\end{abstract}

\section{INTRODUCTION}

It is commonly held that in the case of scalar scattering of a plane wave by a smooth convex body, the contribution by the shadow region may be expressed in terms of "creeping waves" whose attenuation is dependent upon the local radius of curvature (1). For a body whose boundary is a level surface in a coordinate system in which the scalar wave equation is separable, the resolvent theory of linear operators developed by Sims ( 2 ) and Phillips (3) can be applied to obtain a contour integral representation for the distribution. ${ }^{1}$ This integral may then be evaluated by using Langer's theory of asymptotic solutions of differential equations with turning points to compute its residues $(5,6)$. The results obtained are valid in the entire shadow region. We present the details of

* The research reported in this paper has been sponsored by the Air Force Cambridge Research Center, Air Research and Development Command, Contract AF-19(604)-1949.

${ }^{1}$ For a more complete discussion of the reduction of the solution of the time-dependent wave equation to the problem of evaluating a contour integral than is given here see the work of Ritt (4). 
this program in the case of axially-symmetric scalar scattering of a plane wave by a prolate spheroid. We obtain the expected "creeping wave" interpretation of the residue series in which the attenuation of the "creeping waves" depends, in first approximation, on the local radius of curvature. The asymptotic theory used is applicable for large values of $c \omega$, where $2 c$ is the interfocal distance of the spheroid and $\omega$ is the wave number. Thus, our mathematical theory supports the theories of Fock $(7,8)$ and Keller $(1)$, which are based on physical assumptions. The opposite view given in the report (4) is incorrect, and we correct the errors made there here.

The derivation of the above results is made as follows. The point of beginning is the theorem that the well-set exterior problem for the inhomogeneous wave equation $\nabla^{2} u-u_{t t}=\rho e^{i \omega t}$, with boundary conditions on a smooth closed surface and with prescribed initial conditions, has a solution of the form $v(x, t) e^{i \omega t}$ and that its ergodic limit,

$$
\lim _{T \rightarrow \infty} T^{-1} \int_{0}^{T} v(x, t) d t, \text { is } \lim _{s \rightarrow 0} \varphi(x, s)
$$

where $\varphi$ is the solution of the scalar equation $\nabla^{2} \varphi+(\omega-i s)^{2} \varphi=\rho$ satisfying the same boundary conditions as $u$ and which is absolutely square integrable over the region exterior to the boundary surface. The problem is thus reduced to the study of solutions of the last equation. Using the theory of complex resolvents developed by Sims (2) and Phillips (3) a contour integral representation for the desired solution can be obtained in the case where the boundary surface is a level surface of a coordinate system in which the scalar wave equation is separable. This method replaces the use of the Watson transform $(9)$, which has been employed in cylinder and sphere problems $(10,11)$. The integral representation is explicitly determined in the case of the prolate spheroid. This step completes the basic theoretical discussion.

There remains the practical matter of evaluating the contour integral. Because of the assumption of axial symmetry, this integral involves only the Green's functions for the radial and angular parts of the operators into which $\nabla^{2}+$ $(\omega-i s)^{2}$ separates. The integral is evaluated in terms of the residue series generated by the singularities of the radial resolvent Green's function. To determine these singularities, the theory of asymptotic solutions of differential equations having a simple turning point must be used (5). After evaluating the angular resolvent Green's function at these singularities by means of the asymptotic theory for differential equations having a regular singularity and containing a large parameter $(6)$, the values of the residues are obtained. The final stcps involve putting the residue series in proper form for physical interpretation of the characteristics of the solution. In this, the method of Franz (10) is followed. 
2. REDUCTION TO THE SCALAK PROBLEM

We consider the partial differential equation

$$
\nabla^{2} u-u_{t t}=\rho(x) e^{i \omega t},
$$

in which $\rho(x)$ represents a spatial distribution. We seek the solution $u(x, t)$ satisfying homogeneous boundary conditions on a closed, smooth surface $B$ with $u(x, t)$ and $u_{t}(x, t)$ satisfying initial conditions. It is known that the presence of initial conditions prevents the separation of the time dependence from Eq. (2.1). Moreover, the assumption of a "steady state" solution of the well-set problem for Eq. (2.1) is also not in general justifiable. That is, it is not true that a representation of $u(x, t)$ in the form

$$
u(x, t)=u(x) e^{i \omega t}+u^{*}(x, t)
$$

where

$$
\lim _{t \rightarrow \infty} u^{*}(x, t)=0
$$

can be found (12). The following Tauberian type theorem of Ritt (4) clarifies the situation.

Theorem. Let the distribution $\rho(x)$ have compact support, and let $\mathfrak{X}$ be the linear space of twice continuously differentiable functions which satisfy the homogeneous boundary conditions for Eq. (2.1) on $B$ and which vanish at infinity. If $\mathfrak{X}$ is provided with the norm defined by uniform convergence of functions together with their first and second derivatives, then in the scalar wave operator $\nabla^{2}+\omega^{2}$ has a bounded inverse; and the function $v(x, t)$ defined by

$$
v(x, t)=u(x, t) e^{-i \omega t},
$$

where $u(x, t)$ is the solution of the well-set problem for Eq. (2.1), has the C-1 limit $v(x)$,

$$
v(x)=\lim _{T \rightarrow \infty} \frac{1}{T} \int_{0}^{T} v(x, t) d t,
$$

and

$$
\left(\nabla^{2}+\omega^{2}\right) v(x)=\rho(x) .
$$

Now, if $v(x, t)$ is defined as in the theorem, we can show that $v(x, t)$ has a Laplace transform $V(x, s)$ with the following properties:

$$
\begin{gathered}
\int|V(x, s)|^{2} d x<\infty, \\
\lim _{s \rightarrow 0^{+}} s V(x, s)=v(x), \\
{\left[\nabla^{2}+(\omega-i s)^{2}\right][s V(x, s)]=\rho(x) .}
\end{gathered}
$$


In the integral above, the region of integration is the exterior of $B$. We note that the relation (2.3) is an Abelian theorem. Eq. (2.4) can be obtained from Eq. (2.1) after the observation is made that $v(x)$ is independent of the initial conditions. Thus, one may rigorously establish that the solution of the scalar wave equation (2.4), satisfying the condition (2.2), and subjected to the limit process (2.3), yields the (C-1) limit of $u(x, t) \exp (-i \omega t)$. At the same time, the (C-1) limit $v(x)$ obtained satisfies the usual scalar wave equation. It will be seen in the sequel that the meaning of the condition (2.2) is that it ensures that $v(x)$ also satisfies the radiation condition.

Of course, the introduction of a negative imaginary part to the wave number in the scalar wave equation yields a solution in the form of an outgoing wave. We note by the foregoing that a rigorous justification of this accepted procedure comes from the solution of Eq. (2.1) by the Laplace transform method.

\section{SOLUTION OF THE SCALAR WAVE EQUATION}

The preceding argument reduces the well-set problem for Eq. (2.1) to the problem of finding that solution of the elliptic equation

$$
\left[\nabla^{2}+(\omega-i s)^{2}\right] v=\rho \quad(s>0),
$$

which satisfies the homogeneous boundary conditions on $B$ and which is absolutely square integrable over the region exterior to $B$. To proceed further, we now assume that the surface $B$ is described by the equation $\xi=\xi_{0}$, where $(\xi, \eta, \phi)$ is a set of curvilinear coordinates in terms of which the scalar wave equation is separable. These coordinates shall be referred to as the radial, angular, and axial coordinates, respectively. We further assume that $\rho$ has axial symmetry and so remove the dependence on $\phi$ from our consideration. If $\rho$ does not have axial symmetry, the obvious reduction can be made in which Eq. (3.1) is replaced by an equation for the Fourier components of $v$.

We may now write Eq. (3.1) in the form

$$
-L_{\xi} v-L_{\eta} v=J(\xi, \eta) \rho(\xi, \eta) .
$$

In this equation $L_{\xi}$ and $L_{\eta}$ are linear differential operators involving the variables $\xi$ and $\eta$, respectively, and $J(\xi, \eta)$ is the volume Jacobian. Because of the presence of the complex coefficient $(\omega-i s)^{2}$ in $L_{\xi}$ and $L_{\eta}$, these operators are not formally symmetric; and hence, the standard spectral representation theory (13, 14) does not apply. However, such operators have been studied by Sims (2) and Phillips ( 3 ). We next summarize those results of these authors which are applicable to the solution of the boundary value problem for Eq. (3.2).

Let $L$ be a formal differential operator defined by the identity

$$
L y=-\frac{d}{d x}\left\{p(x) \frac{d y}{d x}\right\}+q(x) y \quad(-\infty \leq a<x<b \leq+\infty),
$$


where $p$ is a real-valued differentiable function which is positive on $(a, b)$ and $q$ is a complex-valued function such that $\Im q \geq q_{0}$ on $(a, b)$. There exist integral operators $\mathcal{R}_{\lambda}$, called resolvents for $L$, which are defined and analytic in the half-plane $\Im \lambda<q_{0}$ and which have the property that if $L y$ exists and if $y$ and $L y$ are absolutely square integrable on $(a, b)$, then

$$
\mathcal{R}_{\lambda}(L y-\lambda y)=y
$$

and

$$
\int_{-i \delta-\infty}^{-i \delta+\infty} \Re_{\lambda} y d \lambda=-\pi i y \quad\left(\Im \lambda<q_{0}\right) .
$$

Hereafter, the class of functions $f$ such that $\int_{a}^{b}|f|^{2} d x<\infty$ will be denoted $\mathcal{L}^{2}(a, b)$. In general, $\mathfrak{R}_{\lambda}$ is not uniquely determined. If $q$ does not uniquely determine $Q_{\lambda}$, then the boundary conditions at $a$ and/or $b$ will.

In three dimensional diffraction problems, only two cases are of importance.

Case I. The conditions that $L$ fall into this case are

(a) $-\infty<a$ and $b=\infty$,

(b) $p(a) \neq 0$,

(c) for $3 \lambda<q_{0}$, the homogeneous equation $L y-\lambda y=0$ has exactly one linearly independent solution which is in $\mathscr{L}^{2}(a, \infty)$.

Under these conditions, if a homogeneous boundary condition is set and it is required that $\mathcal{R}_{\lambda} y$ satisfy this condition at $a$ and that $\mathcal{R}_{\lambda} y$ be in $\mathfrak{L}^{2}(a, \infty)$, then $\mathfrak{Q}_{\lambda}$ is uniquely determined and has the representation

$$
\mathfrak{Q}_{\lambda} y=\int_{a}^{\infty} G(x, \tau, \lambda) y(\tau) d \tau
$$

The kernel in this integral is called the resolvent Green's function and is defined by the formula

$$
G(x, \tau, \lambda)=\frac{1}{p(x) W\left(y_{1}, y_{2}, \lambda\right)}\left\{\begin{array}{ll}
y_{1}(x) y_{2}(\tau) & (x<\tau) \\
y_{1}(\tau) y_{2}(x) & (\tau<x)
\end{array},\right.
$$

where $(L-\lambda) y_{j}=0(j=1,2), y_{1}$ satisfies the boundary condition at $a, y_{2}$ is in $\mathcal{L}^{2}(a, \infty)$, and $W\left(y_{1}, y_{2}, \lambda\right)$ is the Wronskian of $y_{1}$ and $y_{2}$ considered as a function of $\tau$.

Case II. The conditions that $L$ fall into this case are

(a) $-\infty<a<b<\infty$,

(b) $a$ and $b$ are regular singular points for $L y-\lambda y=0$, and $p(a)=p(b)=0$.

Here, aside from the condition that $\mathcal{R}_{\lambda} y$ be in $\mathcal{L}^{2}(a, b)$, no boundary conditions are needed in order to specify $\mathfrak{R}_{\lambda}$. The resolvent $\mathfrak{R}_{\lambda}$ again has a representation 
of the form (3.6) (in which, of course, " $\infty$ " is replaced by " $b$ "). In this representation, the kernel is described by the formula (3.7) except that $y_{1}$ and $y_{2}$ are now taken to be the solutions of $L y-\lambda y=0$ which are regular at $a$ and at $b$, respectively.

We now apply these results to obtain the solution of the boundary value problem for Eq. (3.2). In three dimensional diffraction problems, the radial operator $L_{\xi}$, with homogeneous boundary conditions at $\xi_{0}$, satisfies the conditions of Case I above, whereas the angular operator fulfills the conditions of Case II. We thus are able to use the relations (3.4), (3.5), and (3.6) to represent the solution as a contour integral. We give the details of the derivation only in the case of diffraction by a perfectly reflecting prolate spheroid which is considered below. In the case that $B$ is a sphere, this integral representation is preciscly the one which can be obtained by using the Watson transform (10). We carry out the evaluation of the integral, again, only in the case of the prolate spheroid. However, the features the calculation in this example exhibits are typical of the general situation.

\section{THE INTEGRAL REPRESENTATION IN THE PROLATE SPHEROID CASE}

For the prolate spheroid with semifocal distance $c$ and eccentricity $\varepsilon=\xi_{0}^{-1}<$ 1, the operators $L_{\xi}$ and $L_{\eta}$ appearing in Eq. (3.2) are defined by the formulas

$$
\begin{array}{lr}
L_{\xi} u=-\left[\left(\xi^{2}-1\right) u_{\xi}\right]_{\xi}-\gamma^{2}\left(\xi^{2}-1\right) u & \left(\xi_{0}<\xi\right), \\
L_{\eta} u=-\left[\left(1-\eta^{2}\right) u_{\eta}\right]_{\eta}-\gamma^{2}\left(1-\eta^{2}\right) u & (-1<\eta<1)
\end{array}
$$

The constant $\gamma$ appearing above is $c(\omega-i s)$. The boundary condition at $\xi$ must now be specified, and we impose the condition

$$
\left.u_{\xi}\right|_{\xi=\xi_{0}}=0 .
$$

The operators $L_{\xi}$ and $L_{\eta}$ are both of the type (3.3); and for them, respectively,

$$
\begin{aligned}
& \Im q \geq 2 \omega s c^{2}\left(\xi_{0}{ }^{2}-1\right), \\
& \Im q \geq 0 .
\end{aligned}
$$

The radial operator $L_{\xi}$ is to be considered on the interval $\left(\xi_{0}, \infty\right), \xi_{0}>1$. For $L_{\xi}, p=1-\xi^{2}$, and $p\left(\xi_{0}\right) \neq 0$. The homogeneous equation $L_{\xi} y-\lambda y=0$, where $\Im_{\lambda}<2 \omega s c^{2}\left(\xi_{0}^{2}-1\right)$, has two linearly independent solutions $y_{1}(\xi, \lambda)$ and $y_{2}(\xi, \lambda)$ which are asymptotic as $\xi \rightarrow \infty$ to $\left(\xi^{2}-1\right)^{-1 / 2} \exp (i \gamma \xi)$ and $\left(\xi^{2}-1\right)^{-1 / 2}$. $\exp (-i \gamma \xi)$, respectively. Since $\Re(i \gamma)>0$, only the second of these solutions is in $\mathscr{L}^{2}\left(\xi_{0}, \infty\right)$; and hence, $L_{\xi}$ falls into Case I. In order to determine the resolvent Green's function, we must single out the solution satisfying the bound- 
ary condition (4.1) at $\xi_{0}$ and a solution in $\mathscr{L}^{2}\left(\xi_{0}, \infty\right)$. This pair we denote $\varphi_{1}$ and $\varphi_{2}$; and clearly,

$$
\begin{aligned}
& \varphi_{1}(\xi, \lambda)=y_{1}(\xi, \lambda){y_{2}}^{\prime}\left(\xi_{0}, \lambda\right)-y_{2}(\xi, \lambda) y_{1}{ }^{\prime}\left(\xi_{0}, \lambda\right), \\
& \varphi_{2}(\xi, \lambda)=y_{2}(\xi, \lambda) .
\end{aligned}
$$

We note that

$$
\varphi_{1}\left(\xi_{0}, \lambda\right)=\frac{-2 i \gamma}{\xi_{0}^{2}-1} .
$$

The resolvent Green's function is

$$
G\left(\xi, \xi^{\prime}, \lambda\right)=\frac{1}{2 i \gamma \varphi_{2}^{\prime}\left(\xi_{0}, \lambda\right)}\left\{\begin{array}{ll}
\varphi_{1}(\xi, \lambda) \varphi_{2}\left(\xi^{\prime}, \lambda\right) & \left(\xi<\xi^{\prime}\right) \\
\varphi_{2}(\xi, \lambda) \varphi_{1}\left(\xi^{\prime}, \lambda\right) & \left(\xi^{\prime}<\xi\right)
\end{array} .\right.
$$

The operator $L_{\eta}$ is to be considered on $(-1,1)$. One may easily see that it falls into Case II. Its resolvent Green's function is defined in terms of the solution $\psi_{2}(\eta, \mu)$ of $L_{\eta} y-\mu y=0$ which is regular at $\eta=1$ and the solution $\psi_{1}(\eta, \mu)$ defined by $\psi_{1}(\eta, \mu)=\psi_{2}(-\eta, \mu)$. In particular,

$$
\widetilde{G}\left(\eta, \eta^{\prime}, \mu\right)=\frac{1}{\left(1-\eta^{2}\right) \mathscr{W}\left(\psi_{1}, \psi_{2}, \mu\right)}\left\{\begin{array}{ll}
\psi_{1}(\eta, \mu) \psi_{2}\left(\eta^{\prime}, \mu\right) & \left(\eta<\eta^{\prime}\right) \\
\psi_{1}\left(\eta^{\prime}, \mu\right) \psi_{2}(\eta, \mu) & \left(\eta^{\prime}<\eta\right)
\end{array} .\right.
$$

We are now in a position to derive a contour integral representation for the solution $v(\xi, \eta)$ of Eq. (3.2) which we seek. Let $\sigma_{\lambda}$ and $\widetilde{G}_{\mu}$ be the respective resolvents for $L_{\xi}$ and $L_{\eta}$, and let $\Gamma$ be a path in the complex $\lambda$-plane defined by the conditions

$$
\lambda=l+i \delta, \quad 0<\delta<2 \omega s c^{2}\left(\xi_{0}^{2}-1\right),
$$

where $l$ and $\delta$ are real. The orientation of $\Gamma$ is taken so that the direction of increasing $l$ gives positive orientation. We apply $\widetilde{R}_{\lambda}$ and $\widetilde{\mathscr{Q}}_{-\lambda}$ successively to Eq. (3.2), taking into account the relation (3.4) and find

$$
-\tilde{\mathbb{R}}_{-\lambda} v-\mathbb{R}_{\lambda} v=\tilde{R}_{-\lambda} \mathcal{R}_{\lambda}[J \rho] \text {. }
$$

We then integrate both sides of $\mathrm{Eq}$. (4.4) along $\Gamma$ and use the relation (3.5). The following integral representation for $v$ results:

$$
v(\xi, \eta)=\frac{1}{2 \pi i} \int_{\Gamma} \tilde{\mathbb{R}}_{-\lambda} \mathcal{R}_{\lambda}\left[J_{\rho}\right] d \lambda
$$

The justification for this result depends upon the fact that the path $\Gamma$ lies below the singular points of $\mathcal{Q}_{\lambda}$ and above the singular points of $\tilde{R}_{-\lambda}$, a fact guaranteed by the conditions on $\Im_{q}(\mathscr{Z})$. If $s=0$, the argument is invalid. 
To rewrite the integral in the right member of (4.5) we use the representation ensured by formula (3.6). The integrand has the value

$$
\int_{-1}^{+1} \int_{\xi_{0}}^{\infty} \tilde{G}\left(\eta, \eta^{\prime},-\lambda\right) G\left(\xi, \xi^{\prime}, \lambda\right) J\left(\eta^{\prime}, \xi^{\prime}\right) \rho\left(\xi^{\prime}, \eta^{\prime}\right) d \xi^{\prime} d \eta^{\prime} .
$$

Because of our assumption of axial symmetry, we may regard the integral (4.6). as a constant multiple of a volume integral. Then if $\rho(\xi, \eta)$ is a distribution corresponding to a point source at $(\Xi, 1)$, the integrand reduces to

$$
\tilde{G}(\eta, 1,-\lambda) G(\xi, \Xi, \lambda) .
$$

In this case, (4.5) becomes

$$
v(\xi, \eta)=\frac{1}{2 \pi i} \int_{\Gamma} \tilde{G}(\eta, 1,-\lambda) G(\xi, \Xi, \lambda) d \lambda .
$$

When $s \rightarrow 0^{+}$, this reduces to the Green's function for the prolate spheroid relative to a point on the axis of symmetry.

\section{REMARKS ON THE INTEGRAL REPRESENTATION}

Our main concern is to compute the surface distribution on a prolate spheroid induced by an axially-symmetric plane wave. This distribution, $v_{p}\left(\xi_{0}, \eta\right)$, is obtained from $v(\xi, \eta)$ by the standard plane wave normalization, i.e.,

$$
v_{p}\left(\xi_{0}, \eta\right)=\lim _{\Xi \rightarrow \infty}\left(\Xi^{2}-1\right)^{1 / 2} e^{i \epsilon \omega \Xi} \lim _{s \rightarrow 0^{+}} v\left(\xi_{0}, \eta\right) .
$$

Making use of the relations (5.1), (4.7), (4.2), and (4.3) and the asymptotic form of $\varphi_{2}(\Xi, \lambda)$ for $\Xi \rightarrow+\infty$, we obtain the important result that

$$
v_{p}\left(\xi_{0}, \eta\right)=\frac{1}{\left(\xi_{0}^{2}-1\right)} \lim _{s \rightarrow 0^{+}} \frac{1}{2 \pi i} \int_{\Gamma} \frac{\tilde{G}(\eta, 1,-\lambda)}{\varphi_{2}^{\prime}\left(\xi_{0}, \lambda\right)} d \lambda .
$$

The integrand which appears in the relation (5.2) has poles in the half-planes above and below $\Gamma$. These half-planes will hereafter be referred to as the upper and lower half-planes, respectively. The poles lying below $\Gamma$ arise from the singularities of $\widetilde{G}(\eta, 1,-\lambda)$ for fixed $\eta$. The operator $L_{\eta}$ is self-adjoint when $s=0$. Therefore, on the basis of Sturm-Liouville theory we may legitimately evaluate the integral in the relation (5.2) as a residue series involving the residues which arise from the poles of $\widetilde{G}(\eta, 1,-\lambda)$. For large values of $\omega$, the residue series will converge slowly -it is the analog to the expansion in surface harmonics which occurs in the case of the sphere. It thus becomes necessary to consider the residue series contributed by singularities in the upper half-plane. These are precisely the zeros of $\varphi_{2}^{\prime}\left(\xi_{0}, \lambda\right)$. Because the operator $L_{\xi}$, even with $s=0$, is not selfadjoint when the radiation condition is imposed, the question whether or not the integral in the representation (5.2) can be successfully evaluated as a residue 
series contributed by singularities in the upper half-plane is one which can only be settled by considerations removed from Sturm-Liouville theory.

From this point on the broad outline of our work follows that of Franz (10). To determine the residue series, we must obtain a knowledge of the behavior of solutions of the equations

$$
\left[\left(1-\eta^{2}\right) y_{\eta}\right]_{\eta}+\left[\gamma^{2}\left(1-\eta^{2}\right)-\lambda\right] y=0
$$

and

$$
\left[\left(\xi^{2}-1\right) y_{\xi}\right]_{\xi}+\left[\gamma^{2}\left(\xi^{2}-1\right)+\lambda\right] y=0 .
$$

We first consider the contribution to the residue series for the right member of the relation (5.2) when $|\lambda| \gg|\gamma|^{2}$. If $|\lambda| \gg|\gamma|^{2}$, the solutions of Eq. (5.3) behave asymptotically in $\lambda$ like the solutions of Legendre's equation of order $\nu$, where $\nu(\nu+1)=-\lambda$. The resolvent Green's function associated with $L_{\eta}$ is then essentially that obtained in the case of the sphere. A similar consideration applies to Eq. (5.4) but involves slightly more computation.

If we introduce the change of variables defined by

$$
\tilde{y}(\xi)=\left(\xi^{2}-1\right)^{1 / 4} y(\xi) \text { and } z=1 / 2\left[\left(\xi^{2}-1\right)^{1 / 2}+\xi\right],
$$

then Eq. (5.4) becomes

$$
z^{2} \frac{d^{2} \tilde{y}}{d z^{2}}+z \frac{d \tilde{y}}{d z}\left\{\gamma^{2} z^{2}-\left[\frac{1}{2} \gamma^{2}+\frac{1}{4}-\lambda\right]+\frac{1}{4}(z+1 / 4 z)^{-2}\right\} \tilde{y}=0 .
$$

If the last term in the coefficient of $\tilde{y}$ in Eq. (5.5) is suppressed, the equation reduces to Bessel's equation and has solutions $C_{\mu}(\gamma z)$ with $\mu^{2}=1 / 2 \gamma^{2}+1 / 4-\lambda$. Hence, for either fixed $\lambda$ and large $z$ or fixed $z$ and large $\lambda, \gamma^{2}$ being fixed and $|\lambda| \gg|\gamma|^{2}$, the solutions of Eq. (5.5) are asymptotically approximated by cylinder functions $C_{\mu}(\gamma z)$. Because of this fact, the resolvent Green's function associated with $L_{\xi}$ is also closely approximated by the resolvent Green's function in the case of the sphere when $|\lambda| \gg|\gamma|^{2}$. The larger $|\gamma|$ is the better the approximation.

The preceding argument may be formalized so as to show that for $|\lambda| \gg$ $|\gamma|^{2}$, the integrand appearing in the relation (5.2) resembles that obtained in the case of the sphere closely enough that the argument of Franz (Ref. 10, p. 713) may be applied. The conclusion is that in the illuminated region, that is, for positive $\eta$, the residue series diverges; whereas in the shadow region, that is, for negative $\eta$, the residue series converges and represents the solution. This same argument shows that the contribution to the residue series for $|\lambda| \gg|\gamma|^{2}$ may be neglected. To evaluate the integral in the representation (5.2) and give a physical meaning to the result, we must therefore do two things. We shall first compute the residues in the upper half-plane for values of $\lambda$ which are "com- 
parable to" $\gamma^{2}$. We shall then transform the residue series into a double series which, if the order of summation is interchanged, can be interpreted as a series of "creeping waves". We shall see that if the first creeping wave is deleted from this series, the remaining series converges even in the illuminated region. Finally we shall show heuristically that the terms which have been removed can be re-evaluated by the stationary phase technique, and thus find that they correspond to the optical contribution.

\section{THE TURNING-POINT ANALYSIS}

The program we follow to compute the residues contributed by the zeros of $\varphi_{2}^{\prime}\left(\xi_{0}, \lambda\right)$ which lie above the integration path $\Gamma$ is:

(i) Using the theory of Langer (5), we obtain an asymptotic representation for the zeros of $\varphi_{2}^{\prime}\left(\xi_{0}, \lambda\right)$;

(ii) We obtain an asymptotic representation for $d\left[\varphi_{2}^{\prime}\left(\xi_{0}, \lambda\right)\right] / d \lambda$ at these zeros;

(iii) Lastly, we derive an asymptotic representation for $\widetilde{G}(\eta, 1,-\lambda)$ at these zeros, using work of Langer $(6)$. In carrying out $(i)$, we need only to find those zeros which occur for values of $\lambda$ which are comparable in absolute value to $|\gamma|^{2}$. If $|\lambda| \ll|\gamma|^{2}$, we can show that no zeros exist; as we have mentioned above, the zeros corresponding to values of $\lambda$ with $|\lambda| \gg|\gamma|^{2}$ need not be considered.

The homogeneous equation to be studied is Eq. (5.4) with

$$
\xi \geq 1+\epsilon, \epsilon>0, \text { and } \Im \gamma<0 .
$$

If we let

$$
y=\left(\xi^{2}-1\right)^{-1 / 2} w
$$

then

$$
w^{\prime \prime}+\left[\gamma^{2}+\frac{\lambda}{\xi^{2}-1}+\frac{1}{\left(\xi^{2}-1\right)^{2}}\right] w=0,
$$

where the primes denote differentiations with respect to $\xi$. For $|\lambda| \ll|\gamma|^{2}$, this equation has linearly independent solutions $w_{j}(j=1,2)$ such that

$$
w_{j}(\xi, \gamma)=e^{ \pm i \gamma \xi}\left[1+\frac{\mathcal{O}(1)}{\gamma}\right] .
$$

Here and whenever the symbols \pm or $\mp$ appear, the upper sign is to be used when $j=1$, the lower sign when $j=2$. The linearly independent solutions of Eq. (5.4) which are relevant, $y_{j}$, correspond to the functions $w_{j}$ and have the form

$$
y_{j}=\left(\xi^{2}-1\right)^{-1 / 2} e^{ \pm i \gamma \xi}\left[1+\frac{\mathcal{O}(1)}{\gamma}\right] \quad(j=1,2) .
$$


Moreover,

$$
y_{j}^{\prime}=\left( \pm i \gamma-\frac{\xi}{\left(\xi^{2}-1\right)}\right)\left(\xi^{2}-1\right)^{-1 / 2} e^{ \pm i \nu \xi}\left[1+\frac{\mathcal{O}(1)}{\gamma}\right] .
$$

Hence if $|\lambda| \ll|\gamma|^{2}, y_{j}^{\prime}=0$ implies that $\xi /\left(\xi^{2}-1\right)=\mathcal{O}(|\gamma|)$. But if $|\gamma|$ is large and $\xi \geq 1+\epsilon$, this cannot happen; and therefore, since $y_{2}=\varphi_{2}$, if $|\lambda| \ll|\gamma|^{2}, \varphi_{2}^{\prime}\left(\xi_{0}, \lambda\right)$ has no zeros.

For the configuration of $\lambda$ and $\gamma^{2}$ left to be considered, it is convenient, and in fact, is the key to the matter, to introduce an auxiliary parameter $\xi_{1}$ with

$$
\xi_{1} \geq 1+\epsilon \text { and }\left(\xi_{1}^{2}-1\right) \gamma^{2}+\lambda=0 .
$$

For this configuration of $\xi_{1}, \gamma$, and $\lambda$, the differential equation (6.1) has a simple turning point at $\xi_{1}$; and if we eliminate $\lambda$ from the equation using the definition (6.2) of $\xi_{1}$, we obtain the equation

$$
w^{\prime \prime}+\left[\frac{\gamma^{2}\left(\xi^{2}-\xi_{1}{ }^{2}\right)}{\xi^{2}-1}+\frac{1}{\left(\xi^{2}-1\right)^{2}}\right] w=0 .
$$

The solution of this equation whose behavior must be determined is the one asymptotic to $\exp (-i \gamma \xi)$ as $\xi \rightarrow \infty$. Under our hypotheses, the theory of Langer (5) applies to Eq. (6.3), and we may proceed as follows.

Let

$$
U^{2}(\xi)=\frac{\xi^{2}-\xi_{1}^{2}}{\xi^{2}-1},
$$

where $U$ is the branch which is positive for $\xi>\xi_{1}$. The functions of central importance in Langer's theory are:

$$
\Phi(\xi)=\int_{\xi_{1}}^{\xi} U(\tau) d \tau, \quad \zeta(\xi, \gamma)=\gamma \Phi(\xi)
$$

and

$$
\Psi(\xi)=\Phi^{1 / 6} U^{-1 / 2}, \quad \Psi\left(\xi_{1}\right)=\lim _{\xi \rightarrow \xi_{1}} \Psi(\xi) .
$$

With the above definition, $\Psi$ is regular at $\xi_{1}$. We compare solutions of Eq. (6.3) with solutions of the related equation

$$
V^{\prime \prime}+\left[\gamma^{2} U^{2}(\xi)-\frac{\Psi^{\prime \prime}}{\Psi}\right] V=0 .
$$

When $|\zeta| \leq N$, the solutions

$$
V_{j}(\xi)=\Psi \zeta^{1 / 3} J_{\mp 1 / 3}(\zeta)
$$$$
(j=1,2)
$$ 
are convenient to use; while for $|\zeta|>N$, the solutions

$$
V^{(j)}(\xi)=\left(\frac{\pi}{2}\right)^{1 / 2} e^{ \pm(5 \pi i / 12)} \Psi(\xi) \zeta^{1 / 3} H_{1 / 3}^{(j)}(\zeta) \quad(j=1,2)
$$

are convenient to use because of their simple asymptotic behavior in $\zeta$. Here and henceforward " $N$ " is used as a generic symbol for a positive number.

We are interested in the solution $\tilde{w}$ of Eq. (6.3) which is asymptotic to exp $(-i \gamma \xi)$ as $\xi \rightarrow \infty$. Now

$$
V^{(2)}(\xi)=\Psi(\xi) \zeta^{-1 / 6} e^{-i \zeta}\left[1+\mathcal{O}\left(\zeta^{-1}\right)\right] \quad \text { if } \quad|\arg \zeta|<\pi-\delta, \delta>0 ;
$$

and there is a solution $w^{(2)}$ of Eq. (6.3) of the form $V^{(2)}(\xi)\left[1+\mathcal{O}\left(\gamma^{-1}\right)\right]$. Hence it must be that $\tilde{w}$ is a constant multiple of $w^{(2)}$; that is

$$
\tilde{w}=C w^{(2)} \text {, }
$$

where

$$
C=\lim _{\xi \rightarrow \infty} \tilde{w}^{\tilde{w} 2)}=\lambda^{1 / 6} \lim _{\xi \rightarrow \infty} U^{1 / 2} e^{i \gamma[\Phi(\xi)-\xi]} .
$$

To evaluate $C$, it is necessary only to compute

$$
f\left(\xi_{1}\right)=\lim _{\xi \rightarrow \infty}[\Phi(\xi)-\xi],
$$

since

$$
\lim _{\xi \rightarrow \infty} U(\xi)=1 .
$$

To determine $f\left(\xi_{1}\right)$ we let $z=\xi_{1} / \xi$ and use the definitions (6.7), (6.5), and (6.4). We then find

$$
f\left(\xi_{1}\right)=\lim _{z \rightarrow 0}\left\{\xi_{1}^{2} \int_{z}^{1} \frac{1}{t^{2}}\left(\frac{1-t^{2}}{\xi_{1}^{2}-t^{2}}\right)^{1 / 2} d t-\frac{\xi_{1}}{z}\right\} .
$$

An integration by parts yields the formula

$$
f\left(\xi_{1}\right)=\lim _{z \rightarrow 0}\left\{\frac{\left[\left(1-z^{2}\right)\left(\xi_{1}^{2}-z^{2}\right)\right]^{1 / 2}-\xi_{1}}{z}-\int_{z}^{1}\left(\frac{\xi_{1}^{2}-t^{2}}{1-t^{2}}\right)^{1 / 2} d t\right\} .
$$

Finally, we see that

$$
f\left(\xi_{1}\right)=-\int_{0}^{1}\left(\frac{\xi_{1}^{2}-t^{2}}{1-t^{2}}\right)^{1 / 2} d t=-\xi_{1} E\left(\frac{\pi}{2}, \xi_{1}^{-1}\right) .
$$

We observe for future use that

$$
f^{\prime}\left(\xi_{1}\right)=-\xi_{1} \int_{0}^{1}\left[\left(\xi_{1}{ }^{2}-t^{2}\right)\left(1-t^{2}\right)\right]^{-1 / 2} d t=-F\left(\frac{\pi}{2}, \xi_{1}^{-1}\right) .
$$


The functions $F$ and $E$ appearing in these formulas are, respectively, complete elliptic integrals of the first and second kinds.

We can now give the asymptotic representations for $\tilde{w}$ which are guaranteed by Langer's theory. If $\xi \geq 1+\epsilon, \epsilon>0$, and $|\zeta|>N$, then

$$
\tilde{w}=U^{-1 / 2} e^{i\left[\gamma f\left(\xi_{1}\right)-\xi\right]}\left\{1+\frac{B(\xi, \gamma)}{\gamma}+\frac{B(\zeta)}{\zeta}\right\},
$$

and

$$
\tilde{w}^{\prime}=-i \gamma U^{1 / 2} e^{i\left[\gamma f\left(\xi_{1}\right)-\zeta\right]}\left\{1+\frac{B(\xi, \gamma)}{\gamma}+\frac{B(\zeta)}{\zeta}\right\} .
$$

If $\xi \geq 1+\epsilon, \epsilon>0$, and $|\zeta| \leq N$, then

$$
\tilde{w}=\gamma^{1 / 6} e^{i \gamma f\left(\xi_{1}\right)}\left[V^{(2)}(\xi)+\frac{B(\xi, \gamma)}{\gamma}\right]
$$

and

$$
\tilde{w}^{\prime}=\gamma^{1 / 6} e^{i \gamma f(\xi)}\left[V^{(2) \prime}(\xi)+B(\xi, \gamma)\right] .
$$

In these formulas " $B$ " is a generic notation for a function which is bounded with respect to its arguments. The dependence relation

$$
V^{(2)}(\xi)=\gamma_{1} V_{1}(\xi)+\gamma_{2} V_{2}(\xi),
$$

with

$$
\gamma_{1}=i\left(\frac{2 \pi}{3}\right)^{1 / 2} e^{-5 \pi i / 12}, \quad \gamma_{2}=-i\left(\frac{2 \pi}{3}\right)^{1 / 2} e^{-\pi i / 12},
$$

should be used in conjunction with the latter formulas.

$$
\text { 7. THE ZEROS OF } \varphi_{2}{ }^{\prime}\left(\xi_{0}, \lambda\right)
$$

The equality

$$
\varphi_{2}(\xi, \lambda)=\left(\xi^{2}-1\right)^{-1 / 2} \tilde{w}
$$

relates the solution $\varphi_{2}$ of Eq. (5.4) to the solution $\tilde{w}$ of Eq. (6.3). Thus in order to carry out step $(i)$ in the program given in Section 6 , we now find the zeros of

$$
\left(\xi^{2}-1\right)^{1 / 2} \varphi_{2}^{\prime}=\tilde{w}^{\prime}-\frac{\xi}{\xi^{2}-1} \tilde{w},
$$

and in particular, the functional dependence of the zeros of $\varphi_{2}^{\prime}\left(\xi_{0}, \lambda\right)$ on $\lambda$. There are two cases to consider: $|\zeta|>N$ and $|\zeta| \leq N$. 
If $|\zeta|>N$, the relations (6.8) apply; and we can conclude that $\varphi_{2}^{\prime}$ has zeros only if

$$
-i \gamma U^{1 / 2}\left[1+\mathcal{O}\left(\gamma^{-1}\right)\right]-\frac{\xi}{\xi^{2}-1} U^{-1 / 2}\left[1+\mathcal{O}\left(\gamma^{-1}\right)\right]=0,
$$

which implies

$$
\xi=-i \gamma\left[\left(\xi^{2}-1\right)\left(\xi^{2}-\xi_{1}^{2}\right)\right]^{1 / 2}\left[1+\mathcal{O}\left(\gamma^{-1}\right)\right] .
$$

If $\xi>\xi_{1}$, the factor involving $\xi$ and $\xi_{1}$ in the right hand member of this formula is real; hence, $\xi$ must have an imaginary part, contrary to hypothesis. On the other hand, if $\xi<\xi_{1},\left(\xi^{2}-\xi_{1}^{2}\right)^{1 / 2}$ is pure imaginary; and again $\xi$ must have an imaginary part, since $\gamma$ does and $\xi>1$, which is contrary to hypothesis. Thus for unbounded $|\zeta|$, the assumption that $\gamma$ has an imaginary part implies that $\varphi_{2}^{\prime}$ has no zeros.

If $|\zeta| \leq N$, then the relations (6.9) apply. Using them, we find that $\varphi_{2}^{\prime}$ has zeros only where

$$
\left[V^{(2)^{\prime}}+B(\xi, \gamma)\right]-\left[V^{(2)}+B(\xi, \gamma) \gamma^{-1}\right] \xi\left(\xi^{2}-1\right)^{-1}=0 .
$$

When $|\zeta| \leq N, V^{(2)}$ is bounded, and thus $\varphi_{2}^{\prime}$ has zeros only where $\left[V^{(2)^{\prime}}+\right.$ $B(\xi, \gamma)]$ has. If there were a sequence $\left\{\xi_{r}\right\}$ of zeros approaching $\xi_{1}$, then on this sequence, $V^{(2)^{\prime}}$ would remain bounded. But by the relations (6.10) and (6.6),

$$
\lim _{\xi \rightarrow \xi_{1}}\left|V^{(2)^{\prime}}\right|=\infty \text {; }
$$

hence, $\varphi_{2}^{\prime}$ has only a finite number of real zeros $\xi_{r}$ when $|\lambda|$ and $|\gamma|^{2}$ are comparable, and there is an $\epsilon>0$ and an $N>0$ such that

$$
\epsilon<\left|\zeta\left(\xi_{r}\right)\right|<N
$$

for every such $r$. From this last relation and the explicit expression for $V^{(2)}$ which can be obtained from (6.6), it is easy to show that these zeros satisfy an equation of the form

$$
\frac{d}{d \xi}\left[\zeta^{1 / 3} H_{1 / 3}^{(2)}(\zeta)\right]+\mathcal{O}\left(\gamma^{-1}\right)=0 .
$$

If $\xi=\xi_{0}, \xi_{0}$ fixed, and $\lambda$ is considered as variable, the value $\zeta_{r}$ of $\zeta$ corresponding to the $r$ th zero may be thought of as the value of

$$
\zeta=\gamma \int_{\xi_{1}(\lambda, \gamma)}^{\xi_{0}} U\left(t, \xi_{1}\right) d t
$$

which is attained when $\lambda=\lambda_{r}$, since $\xi_{1}$ and $\lambda$ are related by the condition (6.2): that is,

$$
\zeta_{r}=\zeta\left(\xi_{0}, \xi_{1}\left[\lambda_{r}, \gamma\right]\right)
$$


Because the zeros $h_{r}$ of $d\left[t^{1 / 3} H_{1 / 8}^{(2)}(t)\right] / d t$ are simple and this function is analytic in a neighborhood of each of its zeros, the numbers $\zeta_{r}$ determined by the condition (7.2) satisfy the relation

$$
\zeta_{r}=h_{r}+\mathcal{O}\left(\gamma^{-1}\right) .
$$

When considered as a function of $\xi, \zeta^{1 / 3} H_{1 / 3}^{(2)}(\zeta)$ is essentially an Airy function; hence, the known values of the zeros of $A i^{\prime}$ and $A i$ at these zeros may be used to compute $h_{r}$ and $h_{r}{ }^{1 / 3} H_{1 / 3}^{(2)}\left(h_{r}\right)(15)$. Using the definition of $\zeta$, the last relation may be recast in the form

$$
\int_{\xi_{1}\left(\lambda_{r}, \gamma\right)}^{\xi_{0}}\left[\frac{t^{2}-\xi_{1}^{2}\left(\lambda_{r}, \gamma\right)}{t^{2}-1}\right]^{1 / 2} d t=\frac{h_{r}}{\gamma}+\mathcal{O}\left(\gamma^{-2}\right) .
$$

When expanded in powers of $\left(\xi_{0}-\xi_{1}\right)$, the integral on the left becomes

$$
\frac{2}{3}\left(\frac{2 \xi_{0}}{\xi_{0}{ }^{2}-1}\right)^{1 / 2}\left(\xi_{0}-\xi_{1}\right)^{3 / 2}\left[1+\frac{7+\xi_{0}{ }^{2}}{20 \xi_{0}\left(\xi_{0}{ }^{2}-1\right)}\left(\xi_{0}-\xi_{1}\right)+\left(\xi_{0}-\xi_{1}\right)^{2} \mathcal{O}(1)\right] .
$$

For the present, the branch of $\left(\xi_{0}-\xi_{1}\right)^{3 / 2}$ will be left unspecified. Replacing the integral in the relation (7.3) by its equivalent above, we find

$$
\begin{aligned}
\xi_{1}-\xi_{0}=e^{-\pi i / 3}\left(\frac{\xi_{0}{ }^{2}-1}{2 \xi_{0}}\right)^{1 / 3}\left(\frac{3 h_{r}}{2 \gamma}\right)^{2 / 3}\left[1+\frac{e^{-\pi i / 3}\left(7+\xi_{0}{ }^{2}\right)}{10\left(2 \xi_{0}\right)^{4 / 3}\left(\xi_{0}{ }^{2}-1\right)^{2 / 3}}\right. \\
\left.\cdot\left(\frac{2}{3}\right)^{1 / 3}\left(\frac{h_{r}}{\gamma}\right)^{2 / s}+\mathcal{O}\left(\gamma^{-1}\right)\right] .
\end{aligned}
$$

Again we leave the branch of the cube root unspecified.

The numbers $\lambda_{r}$ may now be determined by using the defining relation (6.2) for $\xi_{1}$ in conjunction with the estimate (7.4). The conclusion is

$$
\begin{aligned}
\lambda_{r}=-\gamma^{2}\left(\xi_{0}{ }^{2}-1\right)\left[1+e^{-\pi i / 3}\right. & \left(\frac{2 \xi_{0}}{\xi_{0}{ }^{2}-1}\right)^{2 / 3}\left(\frac{3 h_{r}}{2 \gamma}\right)^{2 / 3}+\frac{4}{5}\left(\frac{3}{2}\right)^{1 / 3} e^{-2 \pi i / 3} \\
& \left.\cdot \frac{2 \xi_{0}{ }^{2}-1}{\left(2 \xi_{0}\right)^{2 / 3}\left(\xi_{0}{ }^{2}-1\right)^{4 / 3}} \cdot\left(\frac{h_{r}}{\gamma}\right)^{4 / 3}+\mathcal{O}\left(\gamma^{-5 / 3}\right)\right] .
\end{aligned}
$$

The branch of the cube root can now be chosen to sutisfy the requirement, obtained from the theory in Section 4, that $\lambda_{r}$ must be in the upper half-plane.

8. THE RESIDUE CONTRIBUTION MADE BY $\varphi_{2}{ }^{\prime}\left(\xi_{0}, \lambda\right)$ AT $\lambda_{r}$

Our next objective is to compute

$$
\left.\frac{\partial \varphi_{2}{ }^{\prime}\left(\xi_{0}, \lambda\right)}{\partial \lambda}\right|_{\lambda=\lambda_{r}} .
$$

Since $\lambda$ and $\xi_{1}$ are related by the formula (6.2), it is sufficient to compute

$$
\left.\frac{\partial \varphi_{2}^{\prime}}{\partial \xi_{1}} \cdot \frac{\partial \xi_{1}}{\partial \lambda}\right|_{\lambda=\lambda_{r}}
$$


where the first factor in this product is to be evaluated at the value of $\xi_{1}$ given by the estimate (7.4). The form of $\varphi_{2}^{\prime}\left(\xi_{0}, \lambda\right)$ as determined by the formulas $(7.1),(6.9)$, and $(6.6)$ is

$$
\begin{aligned}
\varphi_{2}^{\prime}\left(\xi_{0}, \lambda\right)=\left(\xi_{0}{ }^{2}-1\right)^{-1 / 2} \gamma^{1 / 6} e^{i \gamma f\left(\xi_{1}\right)}\left(\frac{\pi}{2}\right)^{1 / 2} e^{-5 \pi i / 12}\left[\left\{\Psi(\xi) \zeta^{1 / 3} H_{1 / 3}^{(2)}(\zeta)\right\}^{\prime}\right. \\
\left.-\frac{\xi}{\xi^{2}-1} \Psi(\xi) \zeta^{1 / 3} H_{1 / 3}^{(2)}(\zeta)+B(\xi, \gamma)\right]_{\xi=\xi_{0}} .
\end{aligned}
$$

From the definitions of $\zeta, \Psi$, and $H_{1 / 3}^{(2)}$ and in conjunction with the estimate (7.4), it may be found by a computation that

$$
\left.\frac{\partial \varphi_{2}{ }^{\prime}\left(\xi_{0}, \lambda\right)}{\partial \xi_{1}}\right|_{\lambda=\lambda r}=\left(\frac{3}{2} \pi \gamma^{3} \xi_{0}\right)^{1 / 2}\left(\xi_{0}{ }^{2}-1\right)^{-1} h_{r} H_{1 / 3}^{(2)}(h r) e^{i \gamma f\left(\xi_{r}\right)+\pi i / 4}\left[1+\mathcal{O}\left(\gamma^{-1 / 3}\right)\right],
$$

where $\xi_{r}$ is $\xi_{1}\left(\lambda_{r}, \gamma\right)$. Because of the critical nature of the phase, the exactness of the term involving $\xi_{r}$ is important. The derivative

$$
\left.\frac{\partial \xi_{1}}{\partial \lambda}\right|_{\lambda=\lambda_{r}}=\frac{-1}{2 \gamma^{2} \xi_{1}\left(\lambda_{r}\right)}
$$

may easily be computed. Thus

$$
\begin{aligned}
& \left.\frac{\partial \varphi_{2}{ }^{\prime}\left(\xi_{0}, \lambda\right)}{\partial \lambda}\right|_{\lambda=\lambda r}=\left(\xi_{0}{ }^{2}-1\right)^{-1}\left\{\frac{3 \pi}{8 \gamma \xi_{0}}\right\}^{1 / 2} h_{r} H_{1 / 3}^{(2)} h_{r} H_{1 / 3}^{(2)}\left(h_{r}\right) e^{i \gamma f\left(\xi_{r}\right)+5 \pi i / 4} \\
& {\left[1+\mathcal{O}\left(\gamma^{-1 / 3}\right)\right]}
\end{aligned}
$$

9. THE DETERMINATION OF $\ddot{G}\left(\eta, 1,-\lambda_{r}\right)$

The final step in our program for the computation of the residues of the integral in the representation (5.2) is to determine an asymptotic representation for $\tilde{G}\left(\eta, 1,-\lambda_{r}\right)$. The differential equation to be considered is now Eq. (5.3) with $-1<\eta<1$ and $\lambda=\lambda_{r}$. Using the theory of Langer (6), we shall derive an asymptotic representation for the solution $\psi_{1}(\eta,-\lambda)$ of Section 4 which is uniform on $-1 \leq \eta \leq 1-\epsilon, \epsilon>0$. The Wronskian $\$\left(\psi_{1}, \psi_{2}\right)$ is a constant multiple of $\left(1-\eta^{2}\right)^{-1}$, and its evaluation is a simple matter.

If we let

$$
y=\left(1-\eta^{2}\right)^{-1 / 2} w \text { and } \rho^{2}=-\lambda,
$$

then since $\lambda_{r}=\left(1-\xi_{r}^{2}\right) \gamma^{2}, \mathrm{Eq}$. (5.3) takes the form

$$
\frac{d^{2} w}{d \eta^{2}}+\left\{\rho^{2}\left[\frac{\xi_{r}^{2}-\eta^{2}}{\left(\xi_{r}^{2}-1\right)\left(1-\eta^{2}\right)}\right]+\frac{1}{\left(1-\eta^{2}\right)^{2}}\right\} w=0
$$

for the values of $\rho$ we need to consider. This equation fulfills the hypotheses necessary to the application of the theory in $(6)$ on $-1<\eta \leq 1$. Following 
Langer, then, we define

$$
P^{2}(\eta)=\frac{\xi_{r}^{2}-\eta^{2}}{\left(\xi_{r}^{2}-1\right)\left(1-\eta^{2}\right)},
$$

with $P$ taken as the positive root,

$$
S(\eta)=\int_{1}^{\eta} P(l) d l, \quad \sigma(\eta, \rho)=\rho S(\eta),
$$

and $M=[P(\eta) S(\eta)]^{-1 / 2}$, with $M(1)=\lim _{\eta \rightarrow 1^{-}} M(\eta)$.

The related equation for (9.2) is that equation of the type

$$
\frac{d^{2} v}{d \eta^{2}}+\left[\rho^{2} P^{2}+\frac{1}{\left(1-\eta^{2}\right)^{2}}+k(\eta)\right] v=0,
$$

which has solutions

$$
v=M \sigma C_{0}(\sigma),
$$

where $C_{0}$ is any cylinder function of order zero. In Eq. (9.3) $k(\eta)$ is a function of the form $\mathcal{O}(1) /(1-\eta)$ on $-1 \leq \eta \leq 1$. Eq. (9.3) is related to Eq. (9.2) in the sense that its solutions are asymptotic representations for solutions of Eq. (9.2). The solutions

$$
v_{0}=M_{\sigma} J_{0}(\sigma)
$$

and

$$
\begin{aligned}
v_{j} & =\left(\frac{\pi}{2}\right)^{1 / 2} e^{ \pm \pi i / 4} M \sigma H_{0}{ }^{(j)}(\sigma), & (j=1,2) \\
& =M \sigma^{1 / 2} e^{ \pm i \sigma}\left[1+\sigma^{-1} \mathcal{O}(1)\right] & (|\sigma| \rightarrow \infty,|\arg \sigma|<\pi-\epsilon),
\end{aligned}
$$

are the ones important to us. Now,

$$
\lim _{\eta \rightarrow 1}\left(1-\eta^{2}\right)^{-1 / 2} v_{0}(\eta)=i \rho,
$$

and $\left(1-\eta^{2}\right)^{-1 / 2} v_{0}$ is regular at $\eta=1$. Only one solution of Eq. (5.3) is regular at 1 ; hence, the theory in $(6)$ guarantees that

$$
\begin{aligned}
\psi_{2}(\eta,-\lambda)= & \frac{1}{i \rho}\left(1-\eta^{2}\right)^{-1 / 2}\left[v_{0}(\eta)+\frac{\sigma^{3} \ln \sigma \mathcal{O}(1)}{\rho^{2}}\right] \quad(|\sigma| \leq N), \\
\psi_{2}(\eta,-\lambda)= & \frac{1}{i \rho}\left(1-\eta^{2}\right)^{-1 / 2}\left\{v_{0}(\eta)+\frac{\sigma^{1 / 2}\left[e^{i \sigma} \mathcal{O}(1)+e^{-i \sigma} \mathcal{O}(1)\right]}{\rho}\right\}(|\sigma|>N), \\
\psi_{2}^{\prime}(\eta,-\lambda)= & \frac{1}{i \rho}\left(1-\eta^{2}\right)^{-1 / 2}\left\{v_{0}^{\prime}+\frac{v_{1}^{\prime} e^{-2 i \sigma} \mathcal{O}(1)+v_{2}^{\prime} e^{+2 i \sigma} \mathcal{O}(1)}{\rho}\right. \\
& \left.\quad+\frac{\eta}{1-\eta^{2}}\left[v_{0}+\frac{\sigma^{1 / 2}\left[e^{i \sigma} \mathcal{O}(1)+e^{-i \sigma} \mathcal{O}(1)\right]}{\rho}\right]\right\}(|\sigma|>M) .
\end{aligned}
$$


As a second solution of Eq. (5.3), we choose

$$
\psi_{1}(\eta,-\lambda)=\psi_{2}(-\eta,-\lambda) \text {. }
$$

The asymptotic behavior of $\psi_{1}$ for $-1 \leq \eta<1$ and of $\psi_{1}{ }^{\prime}$ for $|\sigma(\eta)| \geq N$ may be found from the relations (9.4) and (9.5). As previously noted, the function $\left(1-\eta^{2}\right) W\left(\psi_{1}, \psi_{2}\right)$ is a constant; and hence we may evaluate it at any convenient point. We choose the origin. Since $|\sigma(0, \rho)|>N$,

$$
\begin{aligned}
\left(1-\eta^{2}\right) W\left(\psi_{1}, \psi_{2}\right) & =\frac{2 v_{0}(0) v_{0}^{\prime}(0)}{\rho^{2}}\left[1+\frac{\mathcal{O}(1)}{\rho}\right], \\
& =\left.\frac{1}{\rho} \frac{d\left(v_{0}^{2}\right)}{d \eta}\right|_{\eta=0}\left[1+\frac{\mathcal{O}(1)}{\rho}\right], \\
& =\left\{2 \rho S(\eta) J_{0}(\sigma) J_{0}^{\prime}(\sigma)+\frac{P^{2}-S P^{\prime}}{P^{2}} J_{0}^{2}(\sigma)\right\}_{\eta=0}\left[1+\frac{\mathcal{O}(1)}{\rho}\right] .
\end{aligned}
$$

For $|\sigma|$ large and in particular for $\sigma(0)$, the representations

$J_{0}(\sigma)$

$$
=\left(\frac{2}{\pi \sigma}\right)^{1 / 2}\left\{\cos (\sigma-\pi / 4)\left[1+\mathcal{O}\left(\sigma^{-2}\right)\right]+\sin (\sigma-\pi / 4)\left[\frac{2^{-3}+\mathcal{O}\left(\sigma^{-2}\right)}{\sigma}\right]\right\},
$$

$J_{0}^{\prime}(\sigma)$

$=-\left(\frac{2}{\pi \sigma}\right)^{1 / 2}\left\{\sin (\sigma-\pi / 4)\left[1+\mathcal{O}\left(\sigma^{-2}\right)\right]+\cos (\sigma-\pi / 4)\left[\frac{3 \cdot 2^{-3}+\mathcal{O}\left(\sigma^{-2}\right)}{\sigma}\right]\right\}$

can be used. Therefore,

$$
\left(1-\eta^{2}\right) \operatorname{re}\left(\psi_{1}, \psi_{2}\right)
$$

$$
=\frac{2}{\pi}\left\{\operatorname { c o s } [ 2 \rho S ( 0 ) ] \left[1+\mathcal{O}\left(\sigma^{-2}(0)\right]+\sin [2 \sigma(0)] \mathcal{O}\left(\sigma^{-1}(0)\right\}\left\{1+\frac{\mathcal{O}(1)}{\rho}\right\},\right.\right.
$$

and

$$
\begin{aligned}
\psi_{1}(\eta,-\lambda)=\left\{-i\left(\frac{2}{\pi \rho}\right)^{1 / 2}\left(\frac{\xi_{0}{ }^{2}-1}{\left(\xi_{0}{ }^{2}-\eta^{2}\right)\left(1-\eta^{2}\right)}\right)^{1 / 4}\right. & \cos [\sigma(-\eta)-\pi / 4] \\
& \left.+\rho^{-3 / 2}\left[e^{i \sigma(-\eta)} \mathcal{O}(1)+e^{-i \sigma(-\eta)} \mathcal{O}(1)\right]\right\}
\end{aligned}
$$

for $|\eta| \leq 1-\epsilon$, or $|\sigma(-\eta)| \geq N$.

From these relations, we finally conclude that

$$
\begin{aligned}
\tilde{G}\left(\eta, 1,-\lambda_{r}\right)= & -i\left(\frac{\pi}{2 \rho_{r}}\right)^{1 / 2}\left(\frac{\xi_{0}{ }^{2}-1}{\left(1-\eta^{2}\right)\left(\xi_{0}^{2}-\eta^{2}\right)}\right)^{1 / 4} \\
& \frac{. \cos \left[\rho_{r} S(-\eta)-\pi / 4\right]+\rho_{r}^{-1}\left[e^{i \sigma(-\eta)} \mathcal{O}(1)+e^{-i \sigma(-\eta)} \mathcal{O}(1)\right]}{\left\{\cos \left[2 \rho_{r} S(0)+\rho_{r}^{-1} \sin \left[2 \rho_{r} S(0)\right]\right\}\right.},
\end{aligned}
$$


where

$$
S(\eta)=\frac{-1}{\left(\xi_{r}{ }^{2}-1\right)^{1 / 2}} \int_{\eta}^{1}\left(\frac{\xi_{r}{ }^{2}-t^{2}}{1-t^{2}}\right)^{1 / 2} d t, \quad \rho_{r}{ }^{2}=-\lambda_{r}=\left(\xi_{r}{ }^{2}-1\right) \gamma^{2},
$$

for $|\eta| \leq 1-\epsilon$ or $|\sigma(-\eta)| \geq N$. If $\eta \rightarrow-1$, then $\left|\rho_{r} S(-\eta)\right| \rightarrow 0$; and in this instance, the representation (9.4) with $|\sigma| \leq N$ is to be used. We find that for $|\sigma(-\eta)| \leq N$,

$$
\left\{-i \rho_{r}{ }^{-3 / 2}\left(\frac{\xi_{0}^{2}-1}{\left(\xi_{0}^{2}-\eta^{2}\right)\left(1-\eta^{2}\right)}\right)^{1 / 4}\right.
$$

$\tilde{G}\left(\eta, 1,-\lambda_{r}\right)=\frac{\pi}{2} \cdot \frac{\left.\times S^{1 / 2}(-\eta) J_{0}\left[\rho_{r} S(-\eta)\right]+\frac{\sigma^{3}(-\eta) \ln [\sigma(-\eta)] \mathcal{O}(1)}{\rho_{r}{ }^{3}\left(1-\eta^{2}\right)^{1 / 2}}\right\}}{\left\{\cos \left[2 \rho_{r} S(0)\right]\left[1+\mathcal{O}\left(\sigma^{-2}(0)\right)\right]\right.}$

$$
\left.+\sin [2 \sigma(0)] \Theta\left(\sigma^{-1}(0)\right)\right\}\left\{1+\mathcal{O}(1) \rho_{r}^{-1}\right\}
$$

In the limit as $\eta \rightarrow-1$,

$$
\begin{aligned}
& \widetilde{G}\left(-1,1,-\lambda_{r}\right) \\
& =\frac{\pi}{2} \rho_{r}^{-3 / 2}\left\{\cos \left\{2 \rho_{r} S(0)\right]\left[1+\mathcal{O}\left(\sigma^{-2}(0)\right)\right]+\sin [2 \sigma(0)] \mathcal{O}\left(\sigma^{-1}(0)\right)\right\}^{-1}\left\{1+\frac{\mathcal{O}(1)}{\rho_{r}}\right\} .
\end{aligned}
$$

\section{THE RESIDUE SERIES AND ITS INTERPRETATION}

We now can exhibit the asymptotic form of the residue $R_{r}$ at $\lambda_{r}$ contributed by the right member of the integral representation (5.2). Combining the estimates (9.6), (9.7), and (8.2) and explicitly writing only terms involving the highest powers of $\gamma$, we obtain the result

$$
R_{r} \sim \lim _{s \rightarrow 0^{+}}\left\{\frac{i 2 \cdot 3^{-1 / 2} \xi_{0}{ }^{1 / 2} \exp \left[-i \rho_{r}\left(\xi_{r}{ }^{2}-1\right)^{-1 / 2} f\left(\xi_{r}\right)\right] \cos \left[\rho_{r} S(-\eta)-\pi / 4\right]}{\left[\left(1-\eta^{2}\right)\left(\xi_{0}{ }^{2}-\eta^{2}\right)\right]^{1 / 4} h_{r} H_{1 / 3}^{(2)}\left(h_{r}\right) \cos \left[2 \rho_{r} S(0)\right]}\right\}
$$

when $\left|\rho_{r} S(-\eta)\right|>N$. In this formula, $\xi_{r}$ is to be determined from the relation (7.4) with $\xi_{1}=\xi_{r}$, and $\rho_{r}{ }^{2}=-\lambda_{r}$, where $\lambda_{r}$ is given by the relation (7.5) and $\gamma=\omega-i$ s. If $\left|\rho_{r} S(-\eta)\right| \leq N$, then

$R_{r} \sim \lim _{s \rightarrow 0^{+}}\left\{\frac{i 3^{-1 / 2}\left(2 \pi \xi_{0}\right)^{1 / 2} \exp \left[-i \rho_{r}\left(\xi_{r}^{2}-1\right)^{-1 / 2} f\left(\xi_{r}\right)\right] S^{1 / 2}(-\eta) J_{0}\left[\rho_{r} S(-\eta)\right]}{\gamma\left(\xi_{0}^{2}-1\right)^{1 / 2}\left[\left(1-\eta^{2}\right)\left(\xi_{0}^{2}-\eta^{2}\right)\right]^{1 / 4} h_{r} H_{1 / 3}^{(2)}\left(h_{r}\right) \cos \left[2 \rho_{r} S(0)\right]}\right\}$.

We omit the estimates of error in these results only to avoid excessive complication of already complicated formulas.

By introducing some new parameters, we shall rewrite these results in a form more amenable to interpretation. We first suppose that the limit as $s \rightarrow 0^{+}$has been taken so that $\gamma$ becomes $\varepsilon a \omega$, where $\varepsilon=\xi_{0}{ }^{-1}$ is the eccentricity of the ellipse 
which generates the prolate spheroid and $a$ is its semimajor axis. Of course, $\left(1-\varepsilon^{2}\right)^{1 / 2} a=b$, the semiminor axis. We also let

$$
d(\eta)=b[S(-\eta)-S(0)] \text { and } d^{*}(\eta)=-b[S(-\eta)+S(0)] .
$$

The integral defining $S$ is elliptic; hence, the functions $d$ and $d^{*}$ have the following interpretation. Let a point on a $\xi_{r}$ - spheroid with angular coordinate $\eta$ be considered. Then $|d(\eta)|$ is the length of the shortest geodesic arc from the point to the shadow boundary, the curve on which $\eta=0$. If $-1<\eta<0, d(\eta)>0$; if $0<\eta<1, d(\eta)<0$. The length of that geodesic arc from the point to the shadow boundary which passes through $\eta=-1$ is $d^{*}(\eta)$. Let us also set

$$
\begin{aligned}
\nu_{r}=-\stackrel{\rho}{r}_{b} & \\
=-\omega\left\{1+\frac{1}{2} e^{-\pi i / 3}\left(\frac{3 h_{r}}{\omega b^{2} / a}\right)^{2 / 3}+\right. & e^{-2 \pi i / 3}\left(\frac{h_{r}}{\omega b^{2} / a}\right)^{4 / 3} \\
& \left.\cdot\left[\frac{a^{2}+b^{2}}{5 \cdot 3^{-1 / 3} a^{2}}-3^{4 / 3} \cdot 2^{-3}\right]+\mathcal{O}\left(\omega^{-5 / 3}\right)\right\} .
\end{aligned}
$$

The second of these two equalities is obtained from the relations (9.1) and (7.5). The imaginary part of $\nu_{r}$ is positive. We finally observe that if $L$ denotes the length of the circumference of the ellipse which generates the spheroid, then

$$
L=-4 b S(0) \text {. }
$$

We can now write $R_{r}$ in the forms

$$
R_{r}=A_{r}\left\{\frac{e^{i \boldsymbol{v}_{r} d(\eta)+\pi i / 4}+e^{i \boldsymbol{\nu}_{r} d *(\eta)-\pi i / 4}}{1+e^{i \boldsymbol{r}_{r} L}}\right\} \quad\left(\left|\rho_{r} S(-\eta)\right|>N\right),
$$

and

$$
R_{r}=B_{r}\left\{\frac{e^{i \nu_{r} L / 4}}{1+e^{i \nu_{r} L}}\right\} \quad\left(\left|\rho_{r} S(-\eta)\right| \leq N\right)
$$

where

$$
\left.A_{r}=2 \cdot 3^{-1 / 2} i\left[h_{r} H_{1 / 3}^{(2)}\left(h_{r}\right)\left\{1-\eta^{2}\right)\left(1-\varepsilon^{2} \eta^{2}\right)\right\}^{1 / 4}\right]^{-1},
$$

and

$$
B_{r}=\frac{(2 \pi)^{1 / 2} 3^{-1 / 2} i[d(\eta)-L / 4]^{1 / 2} J_{0}\left[\nu_{r}\left(d^{*}(\eta)-L / 4\right)\right]}{b^{3 / 2} \omega h_{r} H_{1 / 3}^{(2)}\left(h_{r}\right)\left[\left(1-\eta^{2}\right)\left(1-\varepsilon^{2} \eta^{2}\right)\right]^{1 / 4}} .
$$

Of course, the surface distribution $v_{p}=\sum R_{r}$.

If the residues given in Franz's paper (Ref. 10, p. 712) are specialized to the case of a plane wave, and if the relations (10.4), (10.2), and (10.1) are specialized by letting $\varepsilon \rightarrow 0$, then his residues and ours coincide. We must observe, 
however, that since our asymptotic estimates are all made under the hypothesis that $|\gamma|$ is large, and hence that $\varepsilon \omega$ is bounded away from zero, this fact-that the limit as $\varepsilon \rightarrow 0$ of $R_{r}$ as described by relation (10.2) is the known sphere solution-must be regarded as a happy coincidence.

The length $d^{*}(\eta)$ is always positive, and in the shadow region $d(\eta)>0$. Thus the residue series $\sum R_{r}$ for the distribution on the surface converges rapidly in the shadow region and may be summed by the "creeping wave" representation

$$
\sum_{r} R_{r}=\sum_{n=0}^{\infty}(-1)^{n} \sum_{r} A_{r}\left\{e^{i \nu_{r}[d(\eta)+\eta L]+\pi i / 4}+e^{i \nu_{r}\left[d^{*}(\eta)+\eta L\right]-\pi i / 4}\right\} .
$$

【Added in proof: Professor J. B. Keller has shown us that our geometric interpretation of the functions $d(\eta)$ and $d^{*}(\eta)$ is in error, since the $\xi_{r}$-spheroids have no real physical significance. He has shown that since the combination $\rho_{r}\left(\xi_{r}{ }^{2}-1\right)^{-1}$ is identical with $\gamma$, the exponential terms can be correctly interpreted by expanding $i \gamma[S(-\eta)-S(0)]$ and $-i \gamma[S(-\eta)+S(0)]$ as power series in $\left(\xi_{r}-\xi_{0}\right)$ and using the formulas (7.4). This simplifies the result and makes the parameter $\nu_{r}$ and Eq. (10.1) superfluous. We find that the following description of the terms in the expansion can be given.

The leading term is pure imaginary and equals $\omega s$, where $s$ is the arc length from the shadow boundary. The second term is complex and is proportional to $\dot{\omega}^{1 / 3} s^{\prime}$, where $s^{\prime}$ is an integral depending upon the local radius of curvature in exactly the manner predicted by Keller (1). Hence, with this approximation, our theory agrees with the Fock-Keller theory. The next term in the expansion is proportional to $\left(\omega R_{0}\right)^{-1 / 3}$, where $R_{0}$ is the radius of curvature at the tip of the spheroid. Thus, the approximations of Fock and Keller are applicable only when the wave length is small relative to $R_{0}$. We prefer not to speculate on the correctness of the notion of "creeping waves" except to point out that on the basis of Section 2 the integral we have evaluated represents the time average of a timedependent function and not the space-dependent factor of a separated steadystate solution.]

Some remarks on the distribution near the tip in shadow will now be made. The representation (10.3) for $R_{r}$ applies in a neighborhood of the tip whose size depends upon $\rho_{r}$. In first approximation, the region is the one where

$$
\left|\rho_{r}(1+\eta)^{1 / 2}\right|<N \quad\left(\rho_{r} \sim \omega b\right) .
$$

For this configuration of $\rho_{r}$ and $\eta$, the sum $\sum R_{r}$ is rapidly convergent. As $\varepsilon \rightarrow 0^{+}$,

$$
R_{r} \rightarrow \frac{(2 \pi)^{1 / 2} 3^{-1 / 2} i\left[\operatorname{Arcsin}(-\eta)-\frac{\pi}{2}\right]^{1 / 2} J_{0}\left[\rho_{r}\left\{\operatorname{Arcsin}(-\eta)-\frac{\pi}{2}\right\}\right]}{a \omega h_{r} H_{1 / 3}^{(2)}\left(h_{r}\right)\left(1-\eta^{2}\right)^{1 / 4} \cos \left[2 \rho_{r}\left\{\operatorname{Arcsin}(-\eta)-\frac{\pi}{2}\right\}\right]} .
$$


This formula may be of interest even though it is obtained by illegitimate use of our asymptotic representations, since $\gamma=0$ when $\varepsilon=0$.

Lastly, we consider the situation in the illuminated region. We shall argue heuristically to obtain a conclusion analogous to that rigorously obtained in the case of the sphere by Franz (Ref. 10, p. 712). We conjecture that the conclusion is correct and that it can be rigorously proved by following Franz's program. If one rewrites the summation (10.6) as

$$
\sum_{r} R_{r}=\sum_{r} A_{r} e^{i \nu_{r} d(\eta)+\pi i / 4}+\sum^{*},
$$

where $\sum^{*}$ represents the terms for $n=1,2, \cdots$, and the "small" part of the $n=0$ terms, one sees that $\sum^{*}$ represents a series which converges even in the illuminated region. The other terms sum to the integral

$$
\frac{1}{2 \pi i} \int_{\Gamma}\left(\frac{\pi}{2 \rho}\right)^{1 / 2} \frac{\exp \left[-i \rho b^{-1} d(\eta)+\pi i / 4\right]}{\left(\xi_{0}^{2}-1\right) \varphi_{2}{ }^{\prime}\left(\xi_{0}, \lambda\right)} d \lambda .
$$

An examination of Eq. (5.5) reveals that if the quantity $\gamma^{2} z^{2}-\mu^{2}$, where $\mu^{2}=$ $\left(1 / 2 \gamma^{2}+1 / 4-\lambda\right)$, is large in comparison with $(z+1 / 4 z)^{-2}$, the Hankel function representations for $\varphi_{2}^{\prime}\left(\xi_{0}, \lambda\right)$ can be used; hence, one can evaluate the above integral by the method of stationary phase, making use of Langer's representations for Hankel functions. The computation follows that of Franz (Ref. 10, p. 714). The point of stationary phase occurs for the value of $\lambda$ which satisfies the equations

$$
-d(\eta)=\alpha b, \quad \gamma z \cos \alpha=\mu .
$$

But unless $\cos \alpha$ is small or $|d(\eta)|$ is large, these equations contradict the hypothesis that $\gamma^{2} z^{2}-\mu^{2}$ is large. This means that this stationary phase evaluation can be legitimate only in a neighborhood of the specular point of the spheroid; as a matter of fact, the evaluation gives precisely the geometric optics solution.

\section{ACKNOWLedgment}

The authors express their gratitude to R. F. Goodrich for his assistance and faith.

RECEIved: January 5, 1959

\section{REFERENCES}

1. J. B. KeLLER, Trans. I.R.E. AP-4, 312 (1956).

2. A. R. Sims, J. Math. and Mech. 6, 247 (1957).

3. R. S. PhILLIPs, New York University Research Report EM-42 (1952).

4. R. K. Ritt, University of Michigan Report No. 2591-4-T (1958).

5. R. E. Langer, Trans. Am. Math. Soc. 34, 447 (1932).

6. R. E. LANGER, Trans. Am. Math. Soc. 37, 397 (1935).

7. V. A. Fock and M. Leon Tovich, J. Phys. (USSR) 10, 130 (1946).

8. R. F. Goodrich, University of Michigan Report No. 2591-3-T (1958). 
9. G. N. Watson, Proc. Roy. Soc. A95, 83 (1914).

10. W. Franz, Z. Naturforsch. 9a, 705 (1954).

11. I. Imai, Z. Physik 137, 31 (1954).

12. R. M. Lewis, J. Math. and Mech. 7, 593 (1958).

13. M. H. Stone, Am. Math. Soc. Colloq. Publs. No. 15 (1932).

14. E. C. Tiтchmarsh, "Eigenfunction Expansions Associated with Second-Order Differential Equations," Clarendon Press, Oxford, 1946.

15. British Association Mathematical Tables, "The Airy Integral." Cambridge Univ. Press, London and New York, 1946. 